\title{
Gait Analysis using Wearable Sensors with Multiple Sclerosis Patients
}

\author{
Aleksandra JURISIC SKEVIN, Nenad FILIPOVIC, Nikola MIJAILOVIC, Ana DIVJAK, Jasmin NURKOVIC, Radivoje RADAKOVIC, \\ Marija GACIC, Vesna GRBOVIC
}

\begin{abstract}
In this study we investigated gait measurement with wearable sensor for subjects with and without multiple sclerosis (MS) and evaluation gait function.The gait function was measured with Avatar sensors system in 3 patients with MS and in 3 healthy subjects without MS. The system consists of a main sensor node and three additional fixtures. Each sensor node is wearing three-axial accelerometer and two-axis gyroscope. Cross-correlation analysis with the walk signal was applied.Coefficient values from cross-correlation are determined for all 6 subjects. Then for a new unknown subject the cross-correlation was applied and the mean value cross-correlation for healthy subjects was 0.0477 , while in MS subjects this value was 0.0207 . A proven validation for this small training system has shown the evidence for different gait analysis for MS and healthy subjects. This small study opens a new avenue for clinical diagnosis of potential MS subjects while wearable sensor can provide an objective framework for assessing gait abnormality. The measured data can provide better understanding on the progression of the disease and response to treatment.
\end{abstract}

Keywords: cross-correlation coefficient; gait analysis; multiple sclerosis; wearable sensor

\section{INTRODUCTION}

Multiple sclerosis (MS) is a chronic inflammatory disease of the central nervous system, characterized by destruction of axons and neurons. MS is the most common progressive neurological disease in young adults, with a prevalence of $30-110$ per 100000 adults [1-3]. It is believed that in the world there are about 2.3 million people suffering from MS. The incidence of multiple sclerosis in Serbia is estimated at 143/100 000 [4]. MS is characterized by neurological deficits such as motor weakness, spasticity, ataxia and sensory disturbance, and may lead to significant impairment of gait [1-3]. Initial motor symptoms include muscle weakness, hypertonia and coordination problems, which are most frequent in the lower limbs [5]. Balance impairment is common in people with MS and frequently impacts quality of life by decreasing mobility and increasing the risk for falls $[6,7]$. The causes of balance dysfunction in MS are not well understood. More sensitive measures of balance impairment are needed to better understand mechanisms of postural control affected by MS. Dysfunction gait in patients with MS was observed in $85 \%$ of patients as their main problem [8]. A more than one third patients lose the function of walk after 20 years from diagnosis $[9,10]$. For these reasons, it is necessary walking with the supervisory functions in patients with MS. In everyday clinical practice and clinical trials in patients with multiple sclerosis walk and balance evaluation is carried out by EDSS (Expanded Disability Status Scale) score [11].

However, there is no measuring instrument with which to evaluate the gait function in patients. The purpose of this study is to determine if instrumented measures of gait (using wearable sensors) can differentiate people with MS and clinically normal gait from people without MS and evaluation gain function.

\section{METHODS}

We monitored the gait function with the help of Avatar sensor system in 6 subjects where 3 patients were with relapsing-remitting MS and 3 healthy subjects without the disorder treated stationary in the Department of Physical
Medicine and Rehabilitation, Faculty of Medical Science, University of Kragujevac, Serbia, during the year 2016. The control group of 3 subjects was free from any known orthopedic or neurological impairments. The test was approved by the Ethics Committee of the Clinical Center, Kragujevac, Serbia and participants signed consent to participate in the study. Inclusion criteria for patients in the study were: a neurologist-confirmed diagnosis of definite MS according to the revised McDonald criteria [12]; 3.07.0 on the EDSS [11], and relapse-free for at least 30 days prior to testing. Excluding criteria were cognitive decline, impaired vision and hearing, psychiatric disease, orthopedic disorders that could negatively affect mobility, cardiovascular disorders and respiratory disorders. During hospitalization after signing the consent and explaining the objective, the sensor system [13] was applied to the test subjects. Avatar system [13] that was used in the procedure recording is shown in Fig. 1. This system consists of a main sensor node and three additional fixtures. Each sensor node is wearing capacitance based three-axial accelerometer and two-axis gyroscope. The main node communicates with other node using I2C protocol.

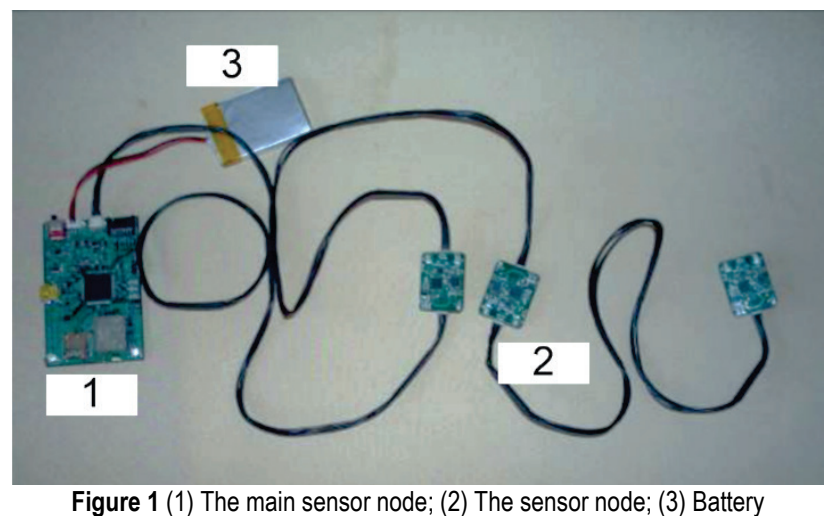

The master node controls network of internal sensor nodes, processes data and communicates with data capture device. The master node features are: ARM7 processor NXP LPC2368 with $58 \mathrm{~KB}$ RAM and $512 \mathrm{~KB}$ program memory and clock rate of $72 \mathrm{MHz}$. 
The whole system is powered by a lithium-polymer battery that provides mobility of the device. From the peripheral sensor nodes data are gather in the main node and sent to a computer via Bluetooth communication. The data are collected, stored and processed in the computer with sampling frequency of $100 \mathrm{~Hz}$. A set signal during the segment of the gait in a patient with a sensor node is shown in Fig. 2.

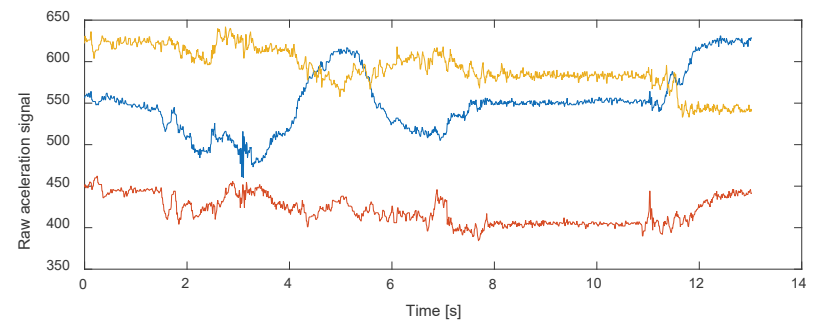

Figure 2 Signal from one acceleration sensor

The signal represents the value of acceleration during gait. The value is hexadecimal number in the range from 0 do 1024 which corresponds to acceleration from $-3 \mathrm{~g}$ to $3 \mathrm{~g}$. We used raw signal from sensors due to reason that only signals shape is relevant for this analysis.

As an indicator of patient mobility can be used mean value of square signals from accelerometers and for each axis separately. This is extremely important because the signal of each axis gives better image and quantification of the movement kinematics than just visual observation. Wang et al 2012 [14] describes a method for the application of cross-correlation analysis with the walk signal. Wren et al 2006 [15] used procedure with the cross-correlation analysis of electromyographic signals during a walk.

\section{EXPERIMENTAL PART}

The measurement of acceleration is performed on the group of six subjects (three healthy and three MS subjects). The position of accelerometer on the subject leg and corresponding axis is shown in Fig. 3.

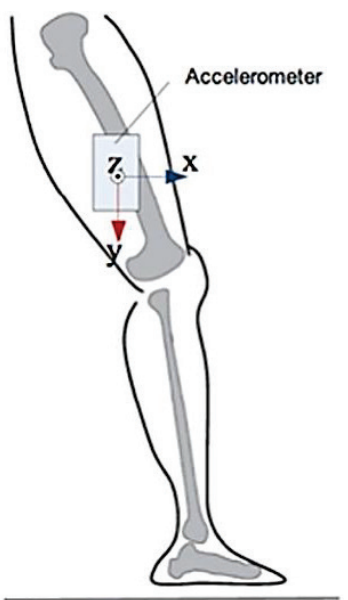

Figure 3 Position of the accelerometer with corresponding axis

The sensors are attached on the leg segment but for analysis only the sensor attached above the knee joint was used. During experiment the subjects are instructed to walk leisurely across pathway 20 meter long and back. All measurements are performed on the room temperature in a period of $175 \mathrm{~s}$.

\section{RESULTS}

If we consider two signals $S(t)$ and $T(t)$ where $t$ is time, the measure of similarity is given by relation

$$
C C=\frac{\sum_{t}(S(t)-\bar{S})(T(t)-\bar{T})}{\sqrt{\sum_{t}(S(t)-\bar{S})^{2} \sum_{t}(T(t)-\bar{T})^{2}}}
$$

where $\bar{S}$ and $\bar{T}$ are mean values of signals $S(t)$ and $T(t)$ respectively. This formula is a cross-correlation coefficient. The coefficient takes the value between -1.0 to 1.0 , while the value closer to 1.0 indicates larger similarity of signals and linear dependency between them. The value of -1 indicates that signals in the opposite phase, which has a linear dependence, but with a negative coefficient. The main idea in this study was using cross-correlation coefficient for classification of signal obtained by accelerometer. We have a signal of accelerometer for healthy and MS subjects. These signals represent training data. For the signal of some new unclassified subject we can calculate cross-correlation coefficient with all training data. The decision of belonging to some group (health subject, subject with disorder) is made according to great value of mean cross-correlation coefficient.
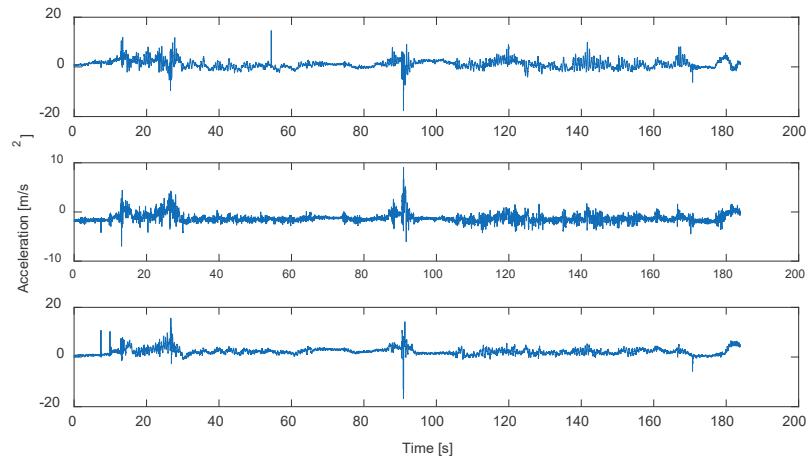

Figure 4 Accelerometer signal for healthy subject
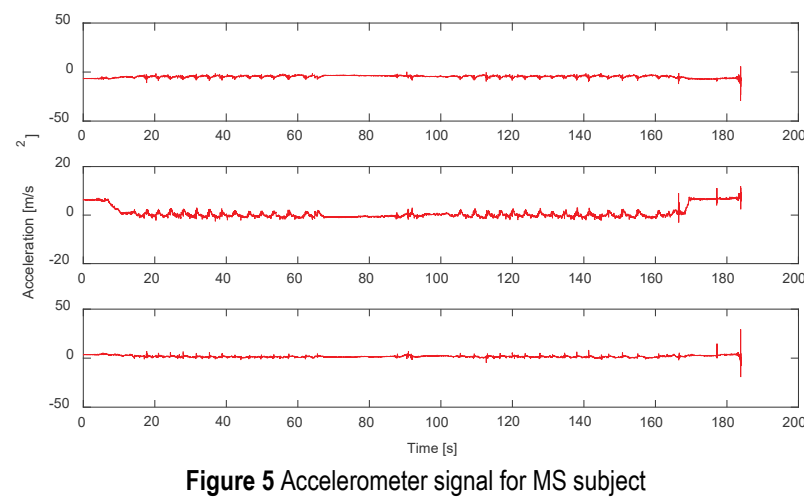

The subject classification according to accelerometer signal of only the $X$ and the $Y$ axis is used while the $\mathrm{Z}$ axis was not considered due to the fact that $Z$ acceleration signal did not significantly vary during gait.

The cross-correlation coefficient for six subjects is shown in Tab. 1 
Table 1 Cross-correlation coefficient for six subjects *- health subject, ${ }^{* *}$ - MS

\begin{tabular}{|c|c|c|c|c|c|c|}
\hline Subject & $1 *$ & $2 *$ & $3^{*}$ & $4^{* *}$ & $5 * *$ & $6^{* *}$ \\
\hline $1 *$ & 1 & 0.078 & 0.054 & 0.021 & 0.037 & 0.041 \\
\hline $2 *$ & 0.078 & 1 & 0.047 & 0.015 & 0.026 & 0.023 \\
\hline $3^{*}$ & 0.054 & 0.047 & 1 & 0.022 & 0.014 & 0.016 \\
\hline $4 * *$ & 0.021 & 0.015 & 0.022 & 1 & 0.042 & 0.051 \\
\hline $5 * *$ & 0.037 & 0.036 & 0.014 & 0.042 & 1 & 0.058 \\
\hline $6 * *$ & 0.041 & 0.023 & 0.016 & 0.051 & 0.058 & 1 \\
\hline
\end{tabular}

In this case, we used only data of acceleration for the $X$-axis. The greater values of cross-correlation coefficients are obtained between subjects of the same group. This result indicated possibility to create database with accelerometer signal, and comparing the cross-correlation coefficient with signals in database can predict MS disorder level of subject during rehabilitation.

The essence of the application of existing methods consists in the fact that the new subjects on the basis of the existing tables determine which group of respondents they belong (healthy or MS disease).

If we have a signal for a new subject (Fig. 6) the idea is to apply the formula for cross-correlation between healthy and MS subjects.

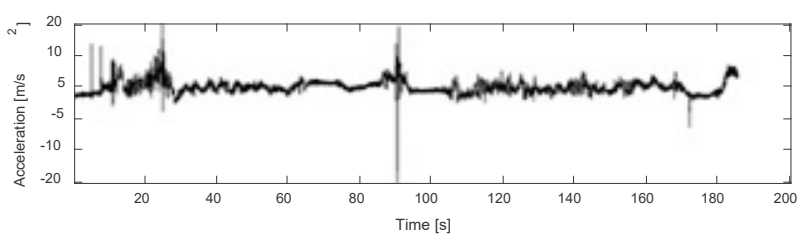

Figure 6 Signal from a new object whose diagnosis is unknown

The formula was applied for cross-correlation between the signals from Fig. 6. For each subject the coefficient values are given in Tab. 2 .

Table 2 Coefficient values from cross-correlation

\begin{tabular}{|c|c|c|c|c|c|c|}
\hline Subject & $1 *$ & $2 *$ & $3 *$ & $4 * *$ & $5 * *$ & $6 * *$ \\
\hline Coefficient value & 0.047 & 0.052 & 0.044 & 0.012 & 0.021 & 0.029 \\
\hline
\end{tabular}

In the healthy subjects, the mean value from crosscorrelation was 0.0477 , while in MS subject this value was 0.0207. Based on these values, we concluded that there was a better similarity between healthy subjects than with the MS subjects so the signal from Fig. 6 corresponds to a healthy subject. It is also validated with standard clinical protocol of study. In the same way, we could carry out a comparison of any signal approximately with similar form as it is given in Tab. 1 and conclude where the subject belongs. It must be considered that the acquisition time signal as well as the trajectory, the moving subjects. To improve this decision system the future aim is to increase the existing base of healthy and diseased subjects, which would be obtained on the reliability of this method of decision-making.

\section{DISCUSSION}

In the patients with MS until now the functionality is accompanied by EDDS score. In these patients there is an objective need for the introduction of new methods for monitoring walk functions. The introduction of sensory systems in everyday clinical practice would be possible for each examination, relapse, therapy change and function of walking for each patient.

It is recommended to evaluate the different possibilities and aspects of physical therapy to be tailored to each patient and for each therapeutic procedure respecting current capabilities of these patients. An important prognostic factor is the movement at the beginning, during and at the end of physical therapy that will allow us, if stagnant, to show that we have reached the current best clinical recovery. Thus, in order to rehabilitate the subjects, the character of these sensors was undeniable. The importance of physical therapy is of great importance in enabling patients to activities of daily living. Feldhege et al. 2015 [16] indicate the clinical significance of using sensors to assess the effectiveness of treatment and the daily work of ambulatory patients with neuromuscular diseases and walk disorders. Also, the study of Solomon et al [17] suggests the clinical relevance of measuring instrumentalized walking in patients with MS using a wireless sensor system performance. Tao and colleagues pointed out the importance and effectiveness of the use of sensors in the assessment of stroke in people with disorders of stroke, in athletes and in rehabilitation [18].

\section{CONCLUSIONS}

The main goal of this study was to use the sensor as a measuring tool in the analysis of gait disturbances in patients suffering from MS so as to have insight into the effectiveness of the therapy applied conservatively and implemented exercise therapy. There is drawback in this study because we consider a small number of subjects, but the results of the assessment of walking are encouraging. The method is painless, with no discomfort for patients. The sensors are easy to apply and the test can be performed easily, quickly and efficiently.

The introduction of wearable sensors into everyday clinical practice would be an adequate indicator of the objective of motor recovery after applied therapeutic modalities. Economically viable sensors would also be important in MS patients monitoring for coming years. There is also possibility to think of monitoring the state of MS patients at home with the help of wearable sensors or using smart phone with accelerometers.

The future research includes creation and use of large database of the accelertion signal in order to create a more reliable decision.

\section{Acknowledgment}

This research is supported by the grants: III41007 and ON174028 Ministry of Education, Science and Technological Development of Serbia.

\section{REFERENCES}

[1] Kraft, A. M. \& Wessman H. C. (1974). Pathology and etiology in multiple sclerosis: a review. Phys Ther., 54, 716720. https://doi.org/10.1093/pt//54.7.716

[2] Matthews, W. B. (1991). Symptoms and signs. In: McAlpine's multiple sclerosis, $2^{\text {nd }}$ ed. New York: Churchill Livingstone, 791-793. 
[3] Shakespeare, D. T., Boggild, M., \& Young, C. (2003). Antispasticity agents for multiple sclerosis. Cochrane Database Syst Rev, 4. https://doi.org/10.1002/14651858.CD001332

[4] Alonso, A. \& Hernan, M. (2008). Temporal trends in the incidence of multiple sclerosis. Neurology, 71, 129-135. https://doi.org/10.1212/01.wnl.0000316802.35974.34

[5] Motl, R. W. \& McAuley, E. (2009). Pathways between physical activity and quality of life in adults with multiple sclerosis. Health Psychol, 28, 682-689. https://doi.org/10.1037/a0015985

[6] Cameron, M. H. \& Huisinga, J. (2014). Objective and subjective measures reflect different aspects of balance in multiple sclerosis. J Rehabil Res Dev., 50(10), 1401-1410. https://doi.org/10.1682/JRRD.2013.02.0042

[7] Cameron, M. H. \& Lord, S. (2010). Postural control in multiple sclerosis: implications for fall prevention. Curr Neurol Neurosci Rep., 10(5), 407-412. https://doi.org/10.1007/s11910-010-0128-0

[8] Scheinberg, L., Holland, N., \& LaRocca, N. G. (1980). Multiple sclerosis; earning a living. NY State J Med., 80, 1395-1400.

[9] Schapiro, R. T. (1991). Multiple sclerosis. A rehabilitation approach to management. New York: Demos.

[10] Weinshenker, B. G., Bass, B., Rice, G. P., Noseworthy, J., Carriere, W., Baskerville, J. et al. (1989). The natural history of multiple sclerosis: a geographically based study. I. Clinical course and disability. Brain, 112, 133-146. https://doi.org/10.1093/brain/112.1.133

[11] Kurtzke, J. F. (1983). Rating neurologic impairment in multiple sclerosis: an Expanded Disability Status Scale (EDSS). Neurology, 33, 1444-1452. https://doi.org/10.1212/WNL.33.11.1444

[12] Polman, C. H., Reingold, S. C., Banwell, B., Clanet, M., Cohen J. A., Fillippi, M. et al. (2011). Diagnostic criteria for multiple sclerosis: 2010 revisions to the McDonald criteria. Ann. Neurol., 69, 292-302. https://doi.org/10.1002/ana.22366

[13] Mijailovic, N., Peulic, A., Filipovic, N., \& Jovanov, E. (2012). Implementation of Wireless Sensor System in Rehabilitation After Back Spine Surgery. Serbian journal of electrical engineering, 9(1), 63-70. https://doi.org/10.2298/SJEE1201063M

[14] Wang, P. \& Wang, J. (2012). Detrended cross-correlation analysis: a new method for gait signal analysis. (Article in Chinese). Journal of Biomedical Engineering, 29(6), 11931196.

[15] Wren, T. A., Do, K. P., Rethlefsen, S.A., \& Healy, B. (2006). Cross-correlation as a method for comparing dynamic electromyography signals during gait. Journal of Biomechanics, 39(14), 2714-2718. https://doi.org/10.1016/j.jbiomech.2005.09.006

[16] Feldhege, F., Mau-Moeller, A., Lindner, T., Hein, A., Markschies, A., Zettl, U. K., \& Bader, R. (2015). Accuracy of a Custom Physical Activity and Knee Angle Measurement Sensor System for Patients with Neuromuscular Disorders and Gait Abnormalities. Sensors, 15, 10734-10752. https://doi.org/10.3390/s150510734

[17] Solomon, A. J., Jacobs, J. V., Lomond, K. V., \& Henry, S. M. (2015). Detection of postural sway abnormalities by wireless inertial sensors in minimally disabled patients with multiple sclerosis: a case-control study. Journal of Neuroengineering and Rehabilitation, 12(74). https://doi.org/10.1186/s12984-015-0066-9

[18] Tao, W., Liu, T., Zheng, R., \& Feng, H. (2012). Gait analysis using wearable sensors. Sensors (Basel), 12(2), 2255-2283. https://doi.org/10.3390/s120202255

\section{Contact information}

Aleksandra JURISIC SKEVIN, PhD, assistant professor Faculty of Medical Science, University of Kragujevac Svetozara Markovica 69, 34000 Kragujevac, Serbia jsaleksandra@gmail.com

Nenad FILIPOVIC, PhD, professor

Faculty of Engineering, University of Kragujevac SestreJanjica 6, 34000 Kragujevac, Serbia BioIRC Research and Development Center for Bioengineering Prvoslava Stojanovica 6, 34000 Kragujevac, Serbia fica@kg.ac.rs

Nikola MIJAILOVIC, PhD student

Faculty of Engineering, University of Kragujevac SestreJanjica 6, 34000 Kragujevac, Serbia

BioIRC Research and Development Center for Bioengineering Prvoslava Stojanovica 6, 34000 Kragujevac, Serbia nmijailo@kg.ac.rs

Ana DIVJAK, PhD student

Faculty of Medical Science, University of Kragujevac Svetozara Markovica 69, 34000 Kragujevac, Serbia anadivjak@gmail.com

Jasmin NURKOVIC, PhD student

Faculty of Medical Science, University of Kragujevac Svetozara Markovica 69, 34000 Kragujevac, Serbia jasmin.nurkovic@gmail.com

Radivoje RADAKOVIC, PhD student

BioIRC Research and Development Center for Bioengineering Prvoslava Stojanovica 6, 34000 Kragujevac, Serbia dididisport@yahoo.com

Marija GACIC, PhD

BioIRC Research and Development Center for Bioengineering Prvoslava Stojanovica 6, 34000 Kragujevac, Serbia marija.gacic@kg.ac.rs

Vesna GRBOVIC, PhD student

Faculty of Medical Science, University of Kragujevac Svetozara Markovica 69, 34000 Kragujevac, Serbia vesna.grbovic@gmail.com 